\title{
How a ball free to orbit in a circular track mitigates the galloping of a square prism
}

Michael Selwanis ( $\triangle$ michael.selwanis@polymtl.ca )

Polytechnique Montréal

\section{Guilherme Rosa Franzini}

Escola politècnica

\section{Cédric Béguin}

Polytechnique Montréal: Polytechnique Montreal

\section{Frédérick P. Gosselin}

Polytechnique Montréal: Polytechnique Montreal

\section{Research Article}

Keywords: Nonlinear energy sink, Ball-in-track (BIT) NES, Vibration suppression, Square prism, Galloping, Energy transfer, Wind tunnel tests, Ball Friction, Power analysis

Posted Date: October 18th, 2021

DOI: https://doi.org/10.21203/rs.3.rs-973095/v1

License: (c) (i) This work is licensed under a Creative Commons Attribution 4.0 International License. Read Full License 


\title{
How a ball free to orbit in a circular track mitigates the galloping of a square prism
}

\author{
Michael M. Selwanis ${ }^{1,2^{*}}$, Guilherme Rosa Franzini $^{3}$, Cédric \\ Béguin $^{1}$ and Frédérick P. Gosselin ${ }^{1 *}$ \\ ${ }^{1 *}$ Department of Mechanical Engineering, Polytechnique \\ Montréal, Québec, Canada. \\ ${ }^{2}$ Aerospace Engineering Department, Military Technical College \\ (MTC), Cairo, Egypt. \\ ${ }^{3}$ Offshore Mechanics Laboratory, Escola Politécnica, University \\ of São Paulo, Brazil.
}

\author{
*Corresponding author(s). E-mail(s): \\ MichaelSelwanis@polymtl.ca; frederick.gosselin@polymtl.ca; \\ Contributing authors: gfranzini@usp.br; \\ cedric.beguin@polymtl.ca;
}

\begin{abstract}
Transverse galloping is a type of flow-induced vibration (FIV) that leads to critical design considerations for engineering structures. A purely nonlinear energy sink (NES) composed of a ball free to rotate in a circular track experimentally mitigated the galloping of a square in a previous study. The current study introduces a model for simulating the dynamics of the square prism coupled with a ball-in-track (BIT) NES and predicting the system behaviour at high flow speeds beyond the limits of the previously presented experiments. Numerical simulations employ the fitting of experimental data as inputs to define parameters. Wind tunnel static experiments provide the galloping force coefficient $\boldsymbol{C}_{\boldsymbol{y}}$ relative to the prism angle of attack. Additionally, free rotation tests allow evaluating the ball damping coefficient $\boldsymbol{c}_{\boldsymbol{\theta}}$ as a function of its mass and the NES track radius. The result of the rotation tests provides a critical angular speed beyond which the ball damping increases nonlinearly. We point out the damping variation as an advantage of the BIT-NES; less damping at low angular velocities helps the ball start its rotation, while relatively large damping at higher speeds dissipates more
\end{abstract}


energy from the vibrating system. Numerical results exhibit four response modes for the NES; oscillatory at low flow speeds, intermittent within a small range of higher flow speeds, rotational at higher flow speeds, and ineffective regime at flow speeds out of the NES effective range. Modelling the primary mass as a parametric excitation source for the NES provides an analytical estimation of the boundary between the oscillatory and intermittent regimes. Furthermore, we advance an analytical analysis of the power flow across the integrated prism-NES system to explain the NES behaviour and predict the limit of its effective range.

Keywords: Nonlinear energy sink, Ball-in-track (BIT) NES, Vibration suppression, Square prism, Galloping, Energy transfer, Wind tunnel tests, Ball Friction, Power analysis.

\section{Introduction}

Nonlinear energy sinks (NESs) represent a specific class of vibration absorbers. A typical NES is composed of a mass attached to the primary system by a spring element of non-linearizable stiffness and appropriate damping properties. The absence of linear natural frequencies enables such absorbers to engage with the dynamics of the primary structure and reduce its vibration amplitudes over a wide range of frequencies (Lee et al, 2008). Purely nonlinear energy sinks are a particular type of these devices with no direct coupling to the primary structure (Pennisi et al, 2017; Li et al, 2018), and interact with the principal vibrating system only due to dynamical interaction. In general, the NES works according to the Targeted Energy Transfer (TET) theory of Gendelman (2008), which is also referred to as energy pumping (Vakakis et al, 2008). This key-concept states that a portion of the energy is transferred irreversibly from the vibrating system to the NES as kinetic energy during the NES motion, reducing the vibration amplitude without a significant change in the system characteristics.

The interest in the area of nonlinear energy absorption has resulted in the development of many designs for NESs. Depending on the NES degree of freedom, we can find two different types; the translative NES that only makes a translational motion (Dongyang et al, 2018; Guo et al, 2017), and the rotative NES (Saeed et al, 2019) composed of a rotating masss. A Vibro-impact NES is a more advanced type (Gendelman and Alloni, 2015) that uses the impact of the NES mass with the walls of the structure as an additional way of energy absorption. The purely rotative NES is a simple and promising device that can damp vibration without a spring element (Ueno and Franzini, 2019).

Following the explanation of the energy transfer phenomena that automatically occurs between a primary linear oscillator and a nonlinear damper 
(Gendelman, 2001), Vakakis (2001) applied the NES to mitigate the response of a structure subjected to an impulse load. Since then, engineers have applied the NES in several applications, including civil engineering structures, mechanical systems, aeronautics and energy harvesting. In the civil engineering field, the NES absorbs the seismic excitation (Menga et al, 2021) of structures, and protects buildings from earthquake loading (Gomez et al, 2021). The NES was presented as a suppressor for structural elements such as beams (Zhang et al, 2020), and plates (Chen et al, 2018). Many studies implemented the NES as a vibration absorber in different mechanical systems; rotary systems (Haris et al, 2020), flywheel (Sun et al, 2018), and self-excited systems (Snoun et al, 2020). Moreover, NESs can suppress the vibrations of helicopter blades (Bergeot et al, 2016). Besides the NES damping effect, integrating such oscillators in piezoelectric (Raj and Santhosh, 2019) or magneto-electric (Jin et al, 2021) systems is an intriguing way for harvesting the energy of a vibrating system.

Concerning the mitigation of flow-induced vibrations, NESs were able to delay the flutter instability of wings (Tian et al, 2019; Amar et al, 2016) and mitigate the oscillations of pipes conveying fluid (Khazaee et al, 2020). NESs of various designs have been implemented in many structures to reduce the impact of flow-induced vibrations. Tumkur et al (2013) computationally simulated the vortex-induced vibration (VIV) of a cylinder coupled with a translative NES, which could reduce the VIV amplitudes by $70 \%$. In a comparable study, Mehmood et al (2014) discussed the effect of initial conditions considered in the simulation on the NES efficiency. Further, Dai et al (2017) computed the fluid loads affecting a cylinder due to VIV using the wake-oscillator models and highlighted the strongly modulated response resulting from the energy transfer with a translative NES. Blanchard et al (2020) adopted computational fluid dynamics (CFD) for estimating the fluid forces and demonstrated that a rotative NES similarly modulates the cylinder dynamics. NES parameters, particularly the mass, affect its capability of reducing the vibration amplitude of the cylinder (Ueno and Franzini, 2019). Recently, Franzini (2021) has used an extensible arm with a tip-mass added to add a radial degree of freedom to the traditional rotative NES, which increased the NES suppression resulting in a $25 \%$ decrease in the maximum amplitude of a cylinder in VIV.

Only a few researchers have introduced the NES as a suppressor for transverse galloping, which is another type of flow-induced vibration generated by heavy winds that can lead to catastrophic effects on high rising structures. A numerical study (Dai et al, 2016) investigated the effect of a translative NES in delaying the galloping of a square prism, demonstrating the influence of NES parameters on its behaviour. In a similar problem, a rotative NES reduced the galloping amplitudes of a prism to $50 \%$ of its original values (Teixeira et al, 2018). The Quasi-steady approach (Parkinson and Smith, 1964) was used for describing the galloping forces. Indeed, square prism galloping involves a hysteresis phenomenon due to a pitchfork bifurcation of the galloping solution within a small range of flow speeds beyond the galloping onset velocity. In 
this parameter range, the prism amplitude depends on the initial conditions; it exhibits a lower limit cycle for increasing flow speed (small initial displacement) and follows the upper branch limit cycle on decreasing flow speed (large initial displacement). In studies using NESs to mitigate galloping, this phenomenon is ignored by considering only small initial displacements for the vibrating prism. The discussion stated that the NES delayed the galloping but did not address the NES ability to absorb the high-amplitude galloping that already occurred. We proposed a purely rotative NES composed of a free ball rotating freely in a circular track to mitigate the galloping of a square prism in a previous study (Selwanis et al, 2021). The rotating ball engaged with the system dynamics and successfully reduced the galloping amplitudes over a wide range of flow speeds in wind tunnel experiments. The limitation of the experimental setup restricted the tests of high-amplitude galloping.

Here we present a mathematical model for the coupled system of the galloping prism and the BIT-NES to simulate its dynamics in a parameter range not easily accessible in experiments. Quasi-static experiments characterise the galloping force coefficient of the prism model $C_{y}$ as a function of the angle of attack. Additionally, we estimate the ball damping coefficient $c_{\theta}$ from the decay rate of the ball angular speed in free rotation tests. The developed model, schematized in Fig. 1, employs the measurements of $C_{y}$ and $c_{\theta}$ as inputs to compute the prism response.

The paper is structured in four sections. Following the introduction, Section 2 presents the proposed model and the evaluation of of each of the galloping force $C_{y}$ and the ball damping coefficients. Additionally, this section demonstrates the experimental setup and the results of both the wind tunnel static experiments and the free rotation tests. Section 3 exhibits the numerical results for the prism response with and without the NES, showing the NES capability in delaying the galloping occurrence and its dynamics at different response modes. In Section 4, we derive an analytical estimation for the limit boundary of the NES oscillatory regime, depending on modelling the primary system mass as a parametric excitation source for the NES. Besides, a power flow analysis of the prism-NES coupled system provides an analytical expectation for the critical flow speed, beyond which the NES can not mitigate high-amplitude galloping. Finally, Section 5 addresses the conclusion.

\section{Mathematical model}

Our model simulates the dynamics of a square prism system coupled with a rotative NES (Fig. 2). We consider an elastically supported square prism of mass $M$, length $l$ and side length $D$ that vibrates transversely to a free stream of velocity $U$ under the effect of the supporting equivalent stiffness $k$, and the structural damping coefficient $c$. A ball of mass $m_{b}$ rotating freely in a circular track of mean radius $r$ represents the rotative NES. The friction between the rotating ball and the NES track results in a ball damping coefficient $c_{\theta}$. The NES ball rotates due to dynamic interaction with the prism vibration reaching 


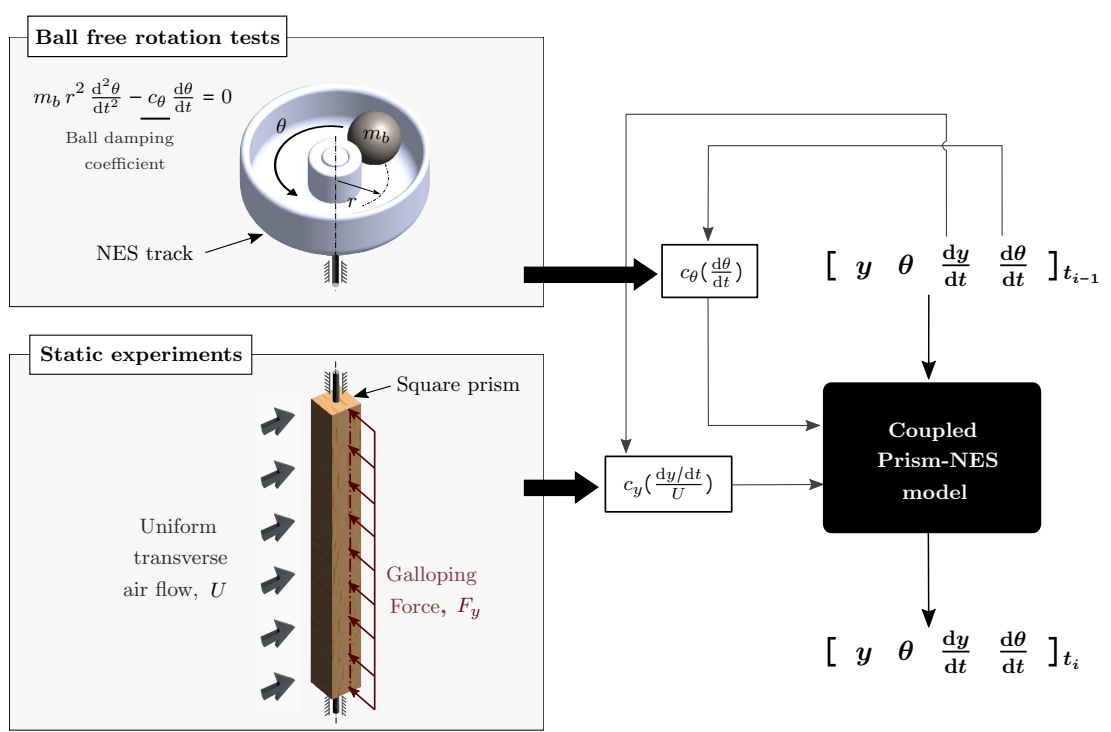

Fig. 1 Diagram showing the ball rotation decay experiments, static wind tunnel experiments and the proposed model that employs these experimental data as inputs to integrate position and velocity of prism $y, \frac{\mathrm{d} y}{\mathrm{~d} t}$ and ball $\theta, \frac{\mathrm{d} \theta}{\mathrm{d} t}$ from time $t_{i-1}$ to time $t_{i}$.

its own angular coordinate $\theta$ (positive in counter-clockwise direction) independently of the prism displacement $y$. Hence, a 2-DOF mathematical model simulates the dynamics of our system; the equation of motion of the vibrating prism in the transverse direction to the flow and ball equation of motion in the tangential direction.

$$
\begin{gathered}
\left(M+m_{b}\right) \frac{\mathrm{d}^{2} y}{\mathrm{~d} t^{2}}+m_{b} r\left(\frac{\mathrm{d}^{2} \theta}{\mathrm{d} t^{2}} \sin \theta+\left(\frac{\mathrm{d} \theta}{\mathrm{d} t}\right)^{2} \cos \theta\right)+c \dot{y}+k y=\frac{1}{2} \rho U^{2} l D C_{y}, \\
m_{b} r^{2} \frac{\mathrm{d}^{2} \theta}{\mathrm{d} t^{2}}+m_{b} r \frac{\mathrm{d}^{2} y}{\mathrm{~d} t^{2}} \sin \theta+c_{\theta} \frac{\mathrm{d} \theta}{\mathrm{d} t}=0 .
\end{gathered}
$$

where $C_{y}$ is the galloping force coefficient, and the natural frequency of the prism is defined as $\omega=\sqrt{\frac{k}{M+m_{b}}}$. To evaluate the parameters of our experimental system, we experimentally estimate the galloping force coefficient $C_{y}$ and the ball damping coefficient $c_{\theta}$, to use them as inputs for the numerical model.

\subsection{Measurement of the galloping force coefficient}

Through static experiments in the closed-loop wind tunnel of the mechanical engineering department of Polytechnique Montréal (Model 407-B, ELD, Lake City, MN, USA), a 6 -axis force/torque balance of $0.0125 \mathrm{~N}$ and 0.001 N.m resolution (GAMMA, ATI Industrial Automation, Apex city, NC, USA) measured the galloping force coefficient of a square prism (Fig. 3). A square prism 


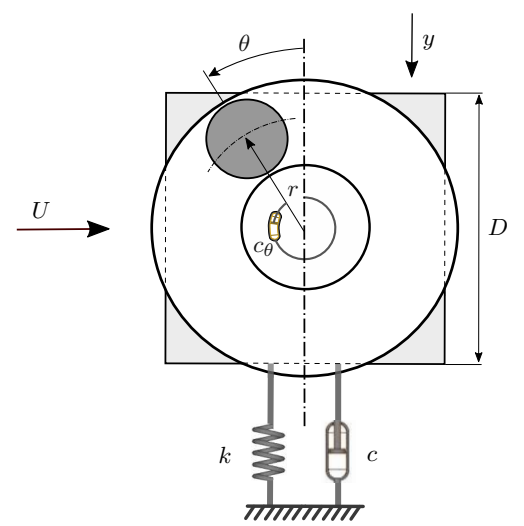

Fig. 2 Schematic drawing of the prism-NES integrated system.

model, Fig. 3a, of length $l$ and side length $D$ made of balsa wood, the same model presented in our previous study (Selwanis et al, 2021), is fixed to the force balance (5) from the upper end. A thin plate of Formica (8) attached to the lower side of the test section supports the other end of the prism preventing any deflection in the X-Y plane. We install two plates (7), of $15 \mathrm{~cm}$ length and $14 \mathrm{~cm}$ width, $8 \mathrm{~cm}$ away from the ends of the prism to help approach twodimensional flow conditions; see Bearman et al (1987). The lengths between the ends of the prism and the supporting are $l_{1}$ and $l_{2}$ at the lower and upper ends, respectively. The force balance rotates with a servo motor (BE231FJNPSN, Parker, Cleveland city, OH, USA) to directly measure the reaction force $R_{y}$ and the moment $M_{x}$ as shown in Fig. 3 a.

Based on the static equilibrium state of the prism (Fig. 3b) and assuming a uniform distributed aerodynamic loading along its length, we compute the galloping force $F_{y}$ from the measured loads $M_{x}$ and $R_{2} . R_{1}$ and $R_{2}$ are the reaction forces in y-direction at the lower and upper ends of the prism, respectively, and $M_{x}$ is the moment measured by the force balance at the upper end of the prism in $\mathrm{x}$-direction.

$$
F_{y}=\left(\frac{l+l_{1}+l_{2}}{l / 2+l_{1}}\right) R_{2}+\left(\frac{1}{l / 2+l_{1}}\right) M_{x}=\frac{1}{2} C_{y}(\alpha) \rho U^{2} l D
$$

where $\rho$ is the flow density, $U$ is the speed of air stream, $l$ is the length of the prism, $D$ is the side length of the square prism, and $C_{y}$ is the galloping force coefficient. We measured $F_{y}$ at different angles of attack $\alpha$, starting from $0^{\circ}$ to $27^{\circ}$ using a step of $3^{\circ}$ in both directions. For each angle, the measurement starts after setting the zero value of the sensor at a stationary flow condition to avoid any residual force with the motor due to rotation. We measure the aerodynamic coefficient at a fixed flow speed $U=30 \mathrm{~m} / \mathrm{s}$ that corresponds to $R e=101 \times 10^{3}$. In each test, two minutes elapsed after reaching the required flow speed to ensure a steady-state. Then, we recorded 150 values of the force 


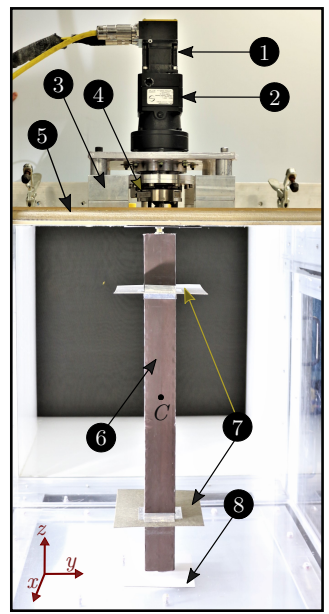

(a)

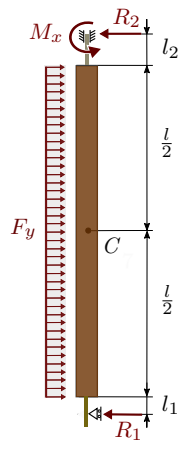

(b)

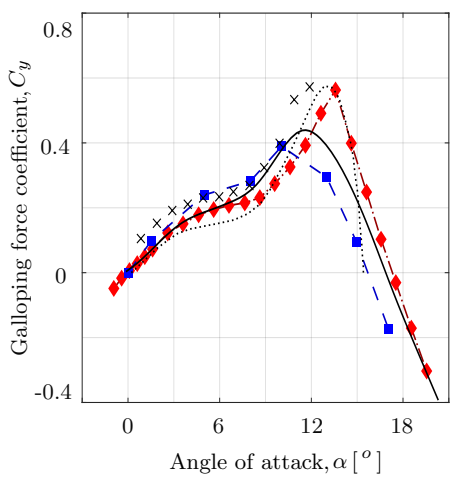

(c)

Fig. 3 (a) Static experiments setup; 1- servo motor, 2- gear reducer, 3- aluminum frame, 4- a wooden panel, 5- Force balance, 6- square prism model, 7- inner plates, 8- outer plates. (b) Free body diagram of the square prism. (c) $C_{y}$ variation with square prism angle of attack $\alpha ;-C_{y}$ curve of the current study at $R e=101 \times 10^{3}$ (see Appx. A for more details), - -Naudascher et al $(1981)$ at $R e=106 \times 10^{3},-\$$-Wawzonek (1979) at $R e=12.4 \times 10^{3}$, .... Parkinson and Smith (1964) at $R e=22.3 \times 10^{3}$, $\times$ Bearman et al (1987) at $R e=14 \times 10^{3}$.

coefficient $C_{y}$ in the consecutive 30 seconds, from which we estimate the average value with a range of fluctuation. Measurement fluctuation was always below $2.5 \%$ for all reported readings. To eliminate any error that may arise from the deviation in the angle measurement, the Kolmogorov-Smirnov (K-S) test compared the $C_{y}$ values measured at the positive and those measured at the negative angles of attack. This statistical method mainly quantifies how the distribution functions of two data samples are similar. A developed MATLAB code shifted the measured $C_{y}$ curves within the uncertainty ranges by $0.65^{\circ}$ to achieve the best symmetry based on the Kolmogorov-Smirnov test, see appendix A. A spline of a smoothing parameter $p=0.999995$ fitted the measured data after eliminating the systematic error representing the final $C_{y}$ curve as a function of the prism angle of attack, $\alpha=(\mathrm{d} y / \mathrm{d} t) / U$.

$$
C_{y}=1.813\left(\frac{\mathrm{d} y / \mathrm{d} t}{U}\right)+24.15\left(\frac{\mathrm{d} y / \mathrm{d} t}{U}\right)^{3}-563.3\left(\frac{\mathrm{d} y / \mathrm{d} t}{U}\right)^{5}+459\left(\frac{\mathrm{d} y / \mathrm{d} t}{U}\right)^{7}
$$

In Fig. 3b, we compare our measurements to some of the previously published data (Parkinson and Smith, 1964; Wawzonek, 1979; Bearman et al, 1987; Naudascher et al, 1981). Comparing to Naudascher et al (1981), the measured $C_{y}$ curve has a small difference of 0.05 in the maximum value of $C_{y}$ and a shift of 2 degrees in the angle of attack corresponding to it. Other measurements at higher Reynolds number $12 \times 10^{3}<R e<23 \times 10^{3}$ show a higher maximum 


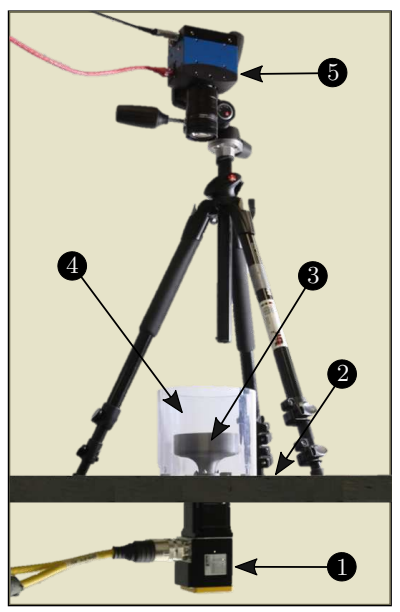

(a)

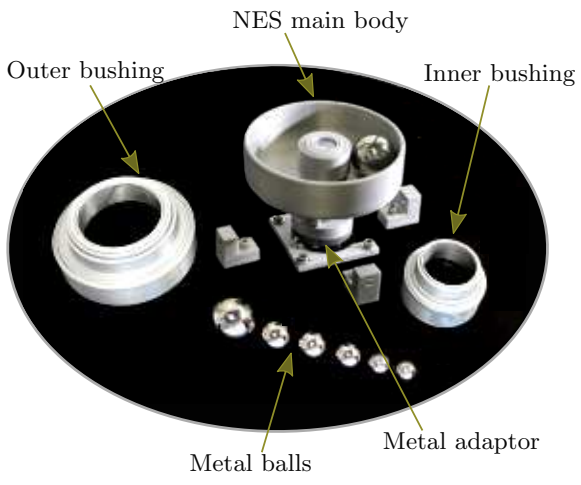

(b)

Fig. 4 Free-rotation tests: (a) Experimental setup; 1- servo motor, 2- test rig, 3- the rotative NES, 4- cylindrical shield, 5- high-speed camera. (b) Exploded view of the NES.

value for $C_{y}$ that corresponds to higher angles of attack. Otherwise, all measurements are close for angles of attack below $12^{\circ}$. Accordingly, we use the $C_{y}$ fitting of our experiments as an input for the numerical model to introduce accurate values for $C_{y}(\alpha)$, presenting the specificity of our model.

\subsection{Representation of the ball damping coefficient}

The ball dynamics is more complicated than that of an attached mass in the traditional rotative NES. The ball behaviour exhibits a combination of sliding and rolling. Characterizing the ball damping is a considerable challenge for the realistic simulation of the prism-NES dynamics. We performed free-rotation tests for estimating the ball damping coefficient. The exponential decay curve of the maximum amplitude represents the damping in a traditional massspring system. For the ball's continuous rotation in our NES, it is convenient to calculate the ball damping from the decay of its angular speed. Since the decay curve varies exponentially, the exponential power used for estimating the damping coefficient does not change.

In the free-rotation tests, a step motor (CM231FJ-115753, Parker) clamped on a wooden plate of a metal-frame test rig rotates the NES track (Fig. 4a). A precise metal adaptor directly connects the motor axis with the NES track to enable fast rotation. For safety requirements, a hollow cylindrical shield of acrylic surrounds the NES to prevent any damages that could result from the ball flying out of the track. We control the angular position, speed and acceleration of the motor to rotate the NES for six complete revolutions at a 50 $\mathrm{rad} / \mathrm{s}$ angular speed. Then, stopping the motor allows the ball to rotate freely, overcoming the friction with the track walls. A high-speed camera captures 
the angular position of the ball with a resolution of $0.2^{\circ}$ to allow for posttreatment image analysis. A developed MATLAB code traces the position of the ball and estimates its average speeds over a revolution $(\mathrm{d} \theta / \mathrm{d} t)_{i}=2 \pi / T_{i}$, where $T_{i}$ is the time taken by the ball to complete the $i^{\text {th }}$ revolution.

Depending on the linear variation of the ball angular positions in the free rotation test (Fig. 5a), we assume a constant ball angular speed (d $\theta / \mathrm{d} t)_{i}$ over each complete revolution. Fig. 5b presents the decay of the angular speed of a $111 \mathrm{~g}$ ball rotating in a circular track of a mean radius $r=30 \mathrm{~mm}$. The ball speed $\dot{\theta}$ decreases rapidly from 40 to $7.7 \mathrm{rad} / \mathrm{s}$, then the rate of decay diminishes for $(\mathrm{d} \theta / \mathrm{d} t)<8 \mathrm{rad} / \mathrm{s}$. Two exponential curves were required for the best fitting of all the estimated values of the ball angular velocity. Each of them provides a ball damping coefficient for a certain angular speed range. The exponential term $e^{b t}$ represents the decay rate of the ball angular speed, where $b=c_{\theta} / 2 m_{b} r^{2}$ based on the equation of motion of a free-rotating ball in the tangential direction

$$
\frac{\mathrm{d}^{2} \theta}{\mathrm{d} t^{2}}+\frac{c_{\theta}}{m_{b} r^{2}} \frac{\mathrm{d} \theta}{\mathrm{d} t}=0 .
$$

The free-rotation test of this NES configuration provides a damping coefficient $c_{\theta 2}=0.00018 \mathrm{~kg} . \mathrm{m}^{2} / \mathrm{s}$ at high ball angular speeds $(\mathrm{d} \theta / \mathrm{d} t)>7.5 \mathrm{rad} / \mathrm{s}$, and a smaller one $c_{\theta 1}=0.000017 \mathrm{~kg} . \mathrm{m}^{2} / \mathrm{s}$ at lower speeds.

The NES design (Fig.4b) allows for changing the width and the mean radius of the track using $3 \mathrm{D}$-printed bushings of small thickness to accommodate different ball sizes. We performed free-rotation tests for many NES configurations. The behaviour observed using the $111 \mathrm{~g}$ ball was a general behaviour observed in the free-rotation tests of other NES configurations, whose results are shown in Table 1 . We fitted each of the two damping coefficients by a spline curve to express it as a function of the ball mass $\left(m_{b}\right)$ and the NES track mean radius $(r)$. Then employ it as an input for the numerical model working with different NES configurations.

$$
\begin{aligned}
c_{\theta_{1}}=( & 0.4+1.7 m_{b}-45.1 r-52.6 m_{b}^{2}+23.9 m_{b} r+1694 r^{2} \\
& \left.+35.6 m_{b}^{3}+1917 m_{b}^{2} r-5483 m_{b} r^{2}-19940 r^{3}\right) \times 10^{-4}, \\
c_{\theta_{2}}=(- & 0.3+0.2 m_{b}+32.2 r-2.4 m_{b}^{2}-15.1 m_{b} r-1232 r^{2} \\
& \left.-52.5 m_{b}^{3}+352.9 m_{b}^{2} r-1818 m_{b} r^{2}+15590 r^{3}\right) \times 10^{-3} .
\end{aligned}
$$

The ball damping experiments come with a critical angular speed $(\mathrm{d} \theta / \mathrm{d} t)_{c}=8 \pm 1.2 \mathrm{rad} / \mathrm{s}$, above which we can not ignore the ball contact with the NES sidewall.At higher angular speeds, the ball contacts two surfaces, the NES flour and the outer sidewall. As a result, it slides and experiences more friction. The friction variation is a considerable advantage for the ball-in-track NES over the traditional rotative NES. Less friction at low speeds helps the 


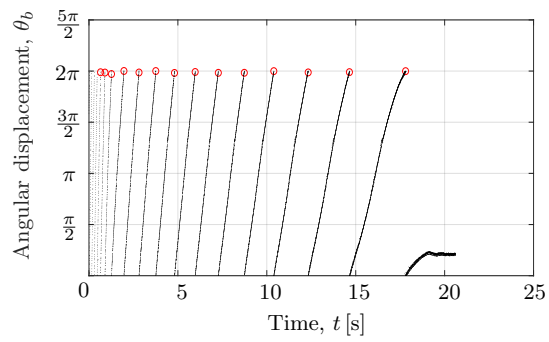

(a)

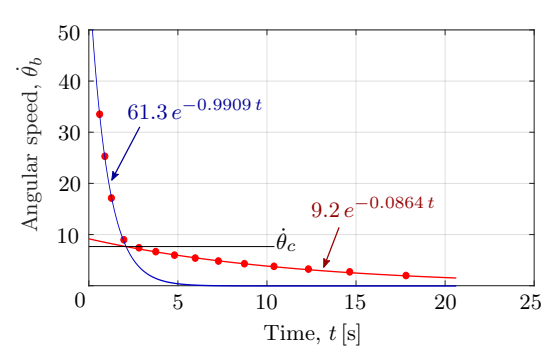

(b)

Fig. 5 Results of the free rotation tests; (a) Ball angular position $v s$ time, marking the end of each revolution (o). (b) The decay in the ball angular speed over each revolution, representing the measured values $(\bullet)$ and the exponential fittings $\left(-61.3 e^{-0.9909 t}\right.$, $\left.-9.2 e^{-0.0864 t}\right)$.

ball to rotate, while the increased friction at high speeds dissipates more energy from the system.

Table 1 Ball damping coefficients of different NES configurations.

\begin{tabular}{|c|c|c|c|c|c|c|c|c|}
\hline \multirow[b]{2}{*}{$r[\mathrm{~m}]$} & \multicolumn{2}{|c|}{$m_{b}=0.014 \mathrm{~kg}$} & \multicolumn{2}{|c|}{$m_{b}=0.014 \mathrm{~kg}$} & \multicolumn{2}{|c|}{$m_{b}=0.056 \mathrm{~kg}$} & \multicolumn{2}{|c|}{$m_{b}=0.111 \mathrm{~kg}$} \\
\hline & $c_{\theta_{1}}$ & $c_{\theta_{2}}$ & $c_{\theta_{1}}$ & $c_{\theta_{2}}$ & $c_{\theta_{1}}$ & $c_{\theta_{2}}$ & $c_{\theta_{1}}$ & $c_{\theta_{2}}$ \\
\hline 0.02 & 2 & 11 & 3.4 & 24 & 5.4 & 44 & & \\
\hline 0.025 & 2.9 & 15 & 4.7 & 34 & 8.3 & 61 & 14.2 & 134 \\
\hline 0.03 & 3.8 & 22 & 7.1 & 48 & 11.3 & 88 & 16.8 & 183 \\
\hline 0.036 & 5.2 & 26 & 8.9 & 55 & 18.7 & 115 & & \\
\hline
\end{tabular}

Note: All the values in the table should be multiplied by $\times 10^{-6}$ to define the ball damping coefficient $c_{\theta}$ in $\mathrm{kg} \cdot \mathrm{m}^{2} / \mathrm{s}$

\subsection{Non-dimensional analysis and Model validation}

For generalisation, we define non-dimensional parameters: non-dimensional vibration displacement $Y$; dimensionless time $\tau$; structural damping ratio $\zeta_{y}$; ball damping ratio $\zeta_{\theta}$; mass ratio (reference air-flow) $m^{*}$; reduced velocity $U_{r}$; NES mass ratio $\hat{m}$ and radius ratio $\hat{r}$ :

$$
\begin{array}{ll}
Y=\frac{y}{D}, & \tau=\omega t, \quad \zeta_{y}=\frac{c}{2\left(M+m_{b}\right) \omega}, \quad \zeta_{\theta}=\frac{c_{\theta}}{2 m_{b} r^{2} \omega}, \\
U_{r}=\frac{U}{\omega D}, \quad m^{*}=\frac{M+m_{b}}{\rho l D^{2}}, \quad \hat{m}=\frac{m_{b}}{M+m_{b}}, \quad \hat{r}=\frac{r}{D} .
\end{array}
$$

Accordingly, the governing equations can be expressed in the non-dimensional form as 


$$
\begin{gathered}
\ddot{Y}+2 \zeta_{y} \dot{Y}+\hat{r} \hat{m}\left(\ddot{\theta} \sin \theta+\dot{\theta}^{2} \cos \theta\right)+Y=\frac{U_{r}^{2}}{2 m^{*}} C_{y}, \\
\ddot{\theta}+2 \zeta_{\theta} \dot{\theta}+\frac{\ddot{Y}}{\hat{r}} \sin \theta=0 .
\end{gathered}
$$

where; $\left({ }^{\prime}\right)$ is the derivative with respect to the dimensionless time $\tau$.

To validate our model, the simulated response of the prism-NES system, as well as the boundaries between NES regimes, are compared with the experiments reported by Selwanis et al (2021). We consider different NES configurations presented in the reference experiments; $\hat{m}=0.08,0.04$ and 0.02 at a constant $\hat{r}$ of 0.6 (Fig. 6a), and $\hat{r}=0.7,0.6$ and 0.5 at a constant $\hat{m}$ of 0.04 (Fig. 6b). All the comparisons are done for low amplitude galloping responses below $\hat{A}=1.5$ due to the experiment's amplitude limitation. The experiments provided measurements at certain flow speeds for the galloping amplitudes and characterized three regimes for the NES, which we present with different marks. While the simulations are presented by continuous lines and the boundaries estimated for each regime are shaded by different colours to exhibit the NES regime mapping.

The prism response curves present the maximum amplitude of the prism versus the reduced flow velocity. At low flow speeds, the prism is stable with the NESs of $\hat{r}=0.6$ (Fig. 6a) until the galloping onset speed $U_{c}=11$, beyond which the prism amplitude jumps to $\hat{A}=0.6$ in the experiments and to 0.4 in the simulations. The NES mitigates the prism vibration delaying the occurrence of large-amplitude galloping to a critical flow speed $U_{\text {crit }}$. The experiments reveal a range for $U_{\text {crit }}$ between the velocity at which the prism amplitude hits the setup limitation $(\hat{A}=1.5)$ and the previously recorded point. The numerical predictions for $U_{\text {crit }}$ agree with the range of experiments for most cases. However, we observe a small deviation between the estimated value and the experiments in the case of $\hat{m}=0.08$ and $\hat{r}=0.6$. Fig. 6 b presents the prism responses with NESs of $\hat{m}=0.04$ and variable $\hat{r}$. Decreasing the NES radius reduces the prism amplitudes and increases $U_{\text {crit }}$. The estimated prism amplitudes show an acceptable deviation compared to the measured values. Regarding the NES regime mapping, the boundaries between the oscillatory and the intermittent regimes predicted by the numerical model match with the experiments in all cases except two: the NES of $\hat{m}=0.02$ and $\hat{r}=0.6$, $U_{\text {crit }}=14$ instead of 16 , and the NES of $\hat{m}=0.04$ and $\hat{r}=0.7, U_{\text {crit }}=15$ instead of 16 . The numerical predictions of the transition between the intermittent and the rotational regimes differ from the experiments only for a NES of $\hat{m}=0.08$ and $\hat{r}=0.6$ and that of $\hat{m}=0.04$ and $\hat{r}=0.4, U_{\text {crit }}=18$ and 16.5 instead of 17 and 20 , respectively.

The simulations predict the same behaviour observed in experiments. Increasing $\hat{m}$ from 0.02 to 0.04 and 0.08 at a constant $\hat{r}=0.6$ expands the NES effective range and widens the range of the intermittent regime. While the decrease of $\hat{r}$ from 0.7 to 0.6 and 0.4 at a constant $\hat{m}=0.04$ reduces the galloping amplitudes, expands the intermittent regime and delays the large-amplitude galloping. 

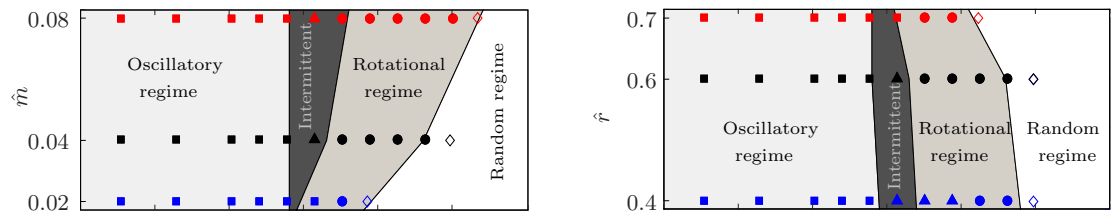

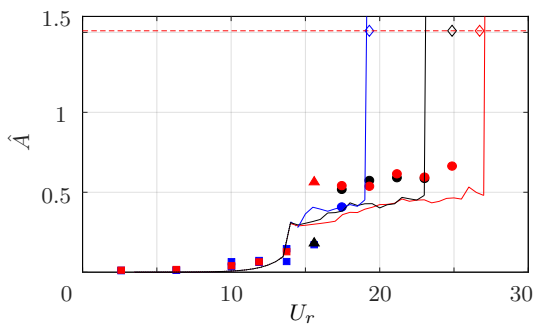

(a)

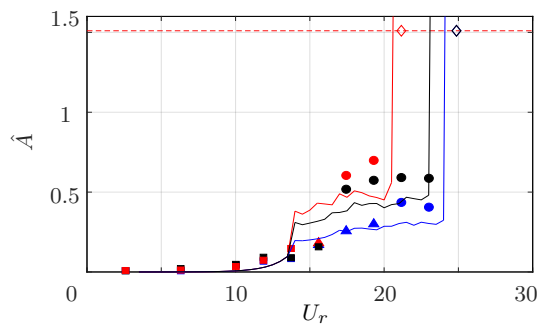

(b)

Fig. 6 Model validation comparing the our simulations for the prism response (continuous curves) and the NES regime mapping with the previous experiments (Selwanis et al, 2021), represented by marks for each of the NES regimes; oscillatory $\mathbf{m}$, Intermittent $\boldsymbol{\Delta}$, and Rotational $\bullet$ regimes, and the points that reach the maximum allowed amplitude $\diamond$. The comparison are done for six various configuration of the NES: (a) $\hat{m}=0.08$ (red), $\hat{m}=0.04$ (black) and $\hat{m}=0.02$ (blue) at constant $\hat{r}=0.6$, (b) $\hat{r}=0.7$ (red), $\hat{r}=0.6$ (black) and $\hat{r}=0.4$ (blue) at constant $\hat{m}=0.04$.

\section{Numerical results}

In the current simulations, ode 45 numerically integrates Eqs. 9 and 10 with a constant time step $\Delta \tau=0.01$ and maximum simulation time $\tau_{\max }=3000$. The selected initial conditions $Y(0)=0.5, \theta(0)=0.1, \dot{Y}(0)=0$ and $\dot{\theta}(0)=0$, lead to zero initial energy of the NES. The characteristic non-dimensional amplitude $\hat{A}$ represents the maximum value of $Y\left(\tau>\tau_{\max } / 3\right)$ to avoid transient response. Our numerical model simulates the dynamics of the prism-NES system at incrementing reduced velocities $3.5<U_{r}<30$, substituting the initial displacement $Y(0)$ of the prism at each flow speed by the maximum amplitude $\hat{A}$ at the previous velocity.

\subsection{NES Effect on galloping response of a prism}

To assess how the NES of mass ratio $\hat{m}=0.08$ and a radius ratio $\hat{r}=0.6$ mitigates the prism galloping, we compared the prism non-dimensional amplitudes $\hat{A}$ with and without the NES in Fig. 7 at different flow reduced velocities. We incremented the reduced flow velocity by a step of 0.5 starting from $U_{r}=3.5$ up to 30 , then decremented it back down with the same velocity step. In the direction of increasing flow speed, the prism amplitude without the NES starts to rise at the galloping onset speed $U_{c}=11.5$, which we determined when the prism non-dimensional amplitude $\hat{A}$ reaches 0.1 . Then, the prism response increases significantly and reaches a lower limit cycle up to a reduced 


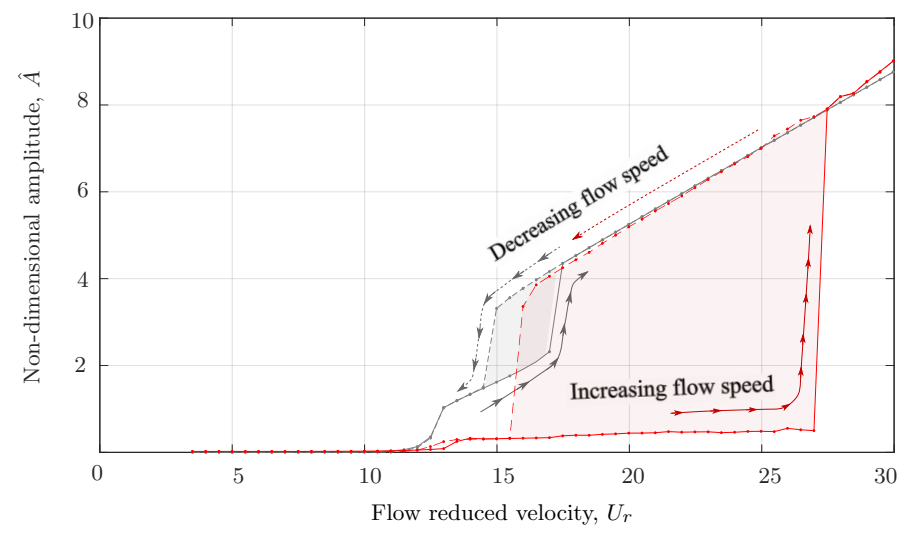

Fig. 7 Numerical simulation of the prism responses with (red curves) and without a NES (gray curves), of a mass ratio $\hat{m}=0.08$, and a radius ratio $\hat{r}=0.6$, in the directions of increasing $U_{r}=3.5: 0.5: 30(-)$ and decreasing flow velocity $U_{r}=30:-0.5: 3.5(--)$.
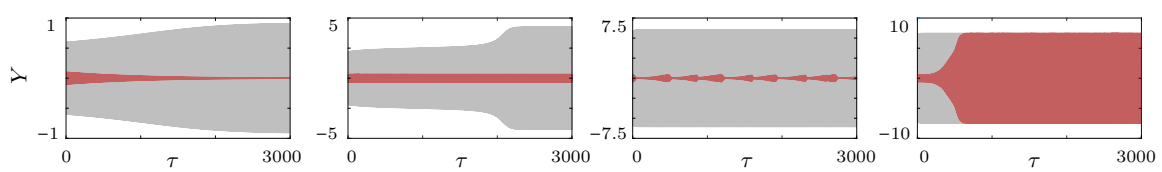

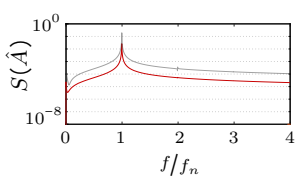

(a)

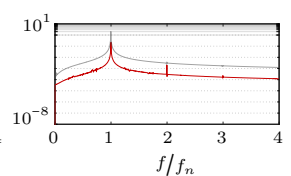

(b)

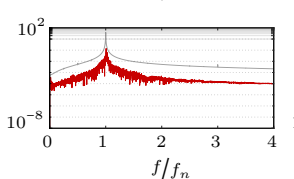

(c)

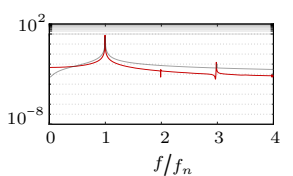

(d)

Fig. 8 The time response and amplitude spectrum of the vibrating prism with (red curve) and without the NES (gray curve), of $\hat{m}=0.08$ and $\hat{r}=0.6$, at different flow reduced velocities $U_{r}=$ : (a) 12.5 , (b) 17.5 , (c) 22.5 , (d) 27.5 . The frequency responses are presented on a semi-log scale.

flow velocity $U_{r}=17.5$. Above this speed, the prism amplitude $\hat{A}$ grows linearly from 4.2 to reach 8.7 at $U_{r}=30$. The NES affects the prism galloping response; it increases the galloping onset speed $U_{c}$ to 13.5 as compared to without NES 11.5. The prism amplitude with the NES does not exceed 0.6 in a wide range of flow speeds $14<U_{r}<27$; which we termed as the NES effective range. At higher flow speeds, the NES is ineffective and may cause an increase in the prism amplitudes. For the prism without NES, we only observed two limit cycle branches between $14<U_{r}<17$. With the NES, the simulation predicts a larger velocity range with two branches $16<U_{r}<27$. For the upper branch (decreasing flow speeds), the NES has no considerable effect on the galloping amplitudes of the prism at high flow speeds $30>U_{r}>16$. At a reduced flow velocity $U_{r}=15.5$, the NES can mitigate the prism galloping and reduces its non-dimensional amplitude $\hat{A}$ from 3.5 down to nearly 0.35 . The 
NES maintains the prism amplitudes around this value for a range of lower speeds $15>U_{r}>13$ and kills the prism vibration at $U_{r}=12$.

Galloping of the square prism considered here involves a hysteresis phenomenon over a range of flow speeds $14.5<U_{r}<17.5$, at which the prism response depends on the initial conditions. With increasing flow speed, the prism follows the lower limit cycle branch. The NES delays the galloping occurrence in this case to much higher flow speeds, mitigating the prism amplitudes in a wide range of flow speeds. With decreasing flow speed, the prism amplitude reaches the upper limit cycle due to the relatively large initial displacement of the prism. The NES is much less effective or ineffective in absorbing highamplitude galloping of the upper branch. Since the NES behaviour depends on the excitation level, the NES is efficient to delay the occurrence of galloping but less effective in stabilizing a prism experiencing very large amplitude galloping.

To evaluate the NES influence on the prism response in the direction of increasing flow speed, we present the displacement time histories and the frequency responses of the prism with and without the NES at reduced flow velocities $U_{r}$ of $12.5,17.5,22.5$, and 27.5 (Fig. 8). While the prism without NES starts to gallop at $U_{r}=12.5$ (Fig. 8a), increasing its non-dimensional displacement $Y$ to 0.9 , the prism-NES system is still stable exhibiting the same natural frequency. At $U_{r}=17.5$ (Fig. 8b), the NES limits the prism amplitude to $\hat{A}=0.3$ in comparison to 4.2 for the prism without NES. At $U_{r}=22.5$ (Fig. 8c), adding the NES reduces the prism amplitude from $\hat{A}=6$ to 0.5 , resulting in a strongly modulated response of the prism. The NES does not change the natural frequency of the prism at $U_{r}=17.5$ and $U_{r}=22.5$. At a reduced flow velocity $U_{r}=27.5$ (Fig. $8 \mathrm{~d}$ ), the NES of mass ratio $\hat{m}=0.08$ is no longer effective in reducing the prism amplitudes. Generally, the NES delays the galloping occurrence and mitigates the prism amplitudes without affecting its natural frequency, even in the NES effective range. That is the main advantage of the NES compared to other vibration absorbers.

\subsection{Different dynamics experienced by the NES}

Since the NES behaviour depends on the excitation level, the change in the galloping force at each flow speed affects the NES dynamics. Based on the NES behaviour, we classify the prism-NES system dynamics into four regimes; oscillatory, intermittent, rotational, and ineffective. The time histories of the prism displacement and the NES ball angular displacement and speed at reduced flow velocities $U_{r}=12.5,15,17.5,25$ and 27.5 exhibit the different dynamics of the system in the four regimes (Fig. 9). Fig. 9a shows the selected points on the prism response curve and presents the NES regime mapping in the two cases of increasing and decreasing the flow velocity.

The oscillatory regime appears at low reduced flow velocities $U_{r}<14$. Figs. $9 \mathrm{~b}$ presents the NES and the prism responses in this regime at $U_{r}=12.5$ : the ball oscillates slowly with a small angular displacement not exceeding $10^{\circ}$, then stops in the $0^{\circ}$ position. The ball angular speed does not exceed $2 \%$ of 
the prism natural frequency. Despite the small oscillations of the NES ball, the NES mitigates the prism amplitudes delaying the occurrence of high-amplitude galloping.

The intermittent regime, characterized by almost steady vibration of the prism (Figs. 9c and 9d), appears as a transition regime existing within a small range of reduced flow velocities $14<U_{r}<18$. The ball oscillates back and forth with an amplitude close to $200^{\circ}$ at the beginning of this regime $U_{r}=15$. While, at the end of this regime $U_{r}=17.5$, the ball behaviour changes to rotate in a few complete revolutions with a low non-dimensional angular speed $\dot{\theta}<0.8$ and thus out of sync with the prism vibrating at a dimensionless frequency of 1 . The NES maintains the prism galloping amplitudes $\hat{A}$ below 0.35 during the whole range of the intermittent regime.

In the rotational regime (Fig. 9e), the NES ball rotates continuously for more than 5 revolutions with higher angular speed $\dot{\theta}$ that fluctuates around 1, i.e., approximately equal to the prism frequency. The ball rotation directly reduces the galloping amplitudes from 0.5 to 0.05 before stopping. Then the prism amplitude grows again. This cycle of reduction and growing of the prism amplitude is repeated, resulting in a strongly modulated response of the vibrating prism.

At higher flow speeds $U_{r} \geq 27.5$, the ineffective regime (Fig. 9f) appears, in which the ball can not engage with the system frequency at high amplitude galloping. Thus, the ball rotates, alternating its directions with high angular speeds approaching 4 times the prism natural angular frequency. These fast rotations of the ball are insufficient to damp the prism motion. In the ineffective regime, the NES is no longer beneficial in mitigating the galloping amplitude at high flow speeds out of its effective range as the energy extracted by the vibrating prism from the airflow exceeds the energy dissipation rate of the NES.

Upon slowing down the flow from $U_{r}=30$, the prism-NES system experiences an ineffective regime for $U_{r} \geq 27.5$. Then the system behaviour changes at $U_{r}=25$ and 17.5 (Figs. 9 e' and d', respectively). The NES ball experiences ineffective rotation at $U_{r}=25$, which changes to large oscillations at 17.5 with no damping effect on the prism amplitudes. At $U_{r}<16$, the NES can damp the galloping of the prism, reducing its non-dimensional amplitude from $\hat{A}=3.8$ to 0.3 (Figs. 9 a). We observe the NES intermittent response when slowing down the flow in a narrow range of reduced flow speeds $14<U_{r}<16$. While, the oscillatory regime appears at the same flow speed range $U_{r}<14$, comparing to the case of accelerating flow.

We confirm that the NES response depends on the excitation level of the primary system. In the oscillatory regime, the kinetic energy consumed to oscillate the NES ball with small oscillations is enough to absorb the energy extracted from the flow by the prism at low flow speeds and delay the galloping occurrence. When the energy extracted by the prism from the air stream increases at higher flow speeds, the balls rotates fast in the intermittent regime and faster in the rotational regime to absorb the prism energy. According to 


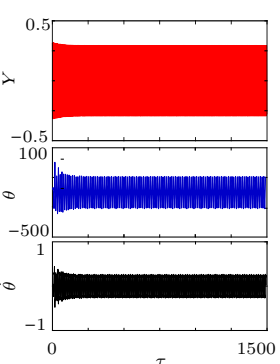

(c)

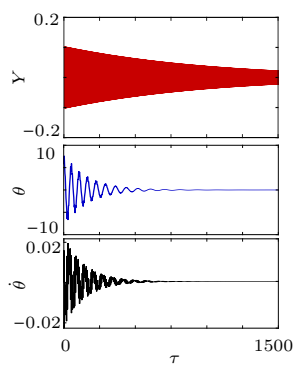

(b)
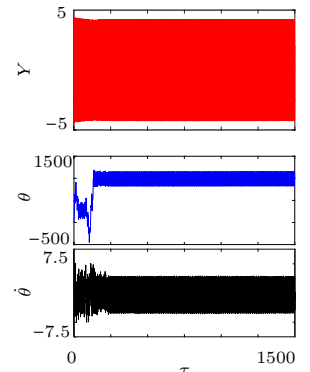

(d')

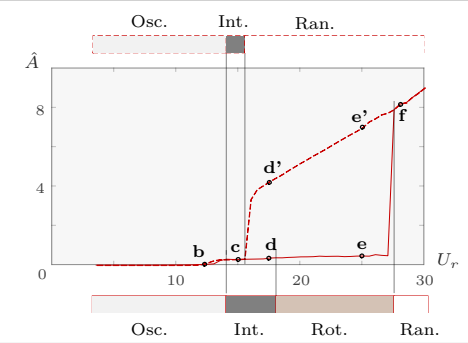

(a)
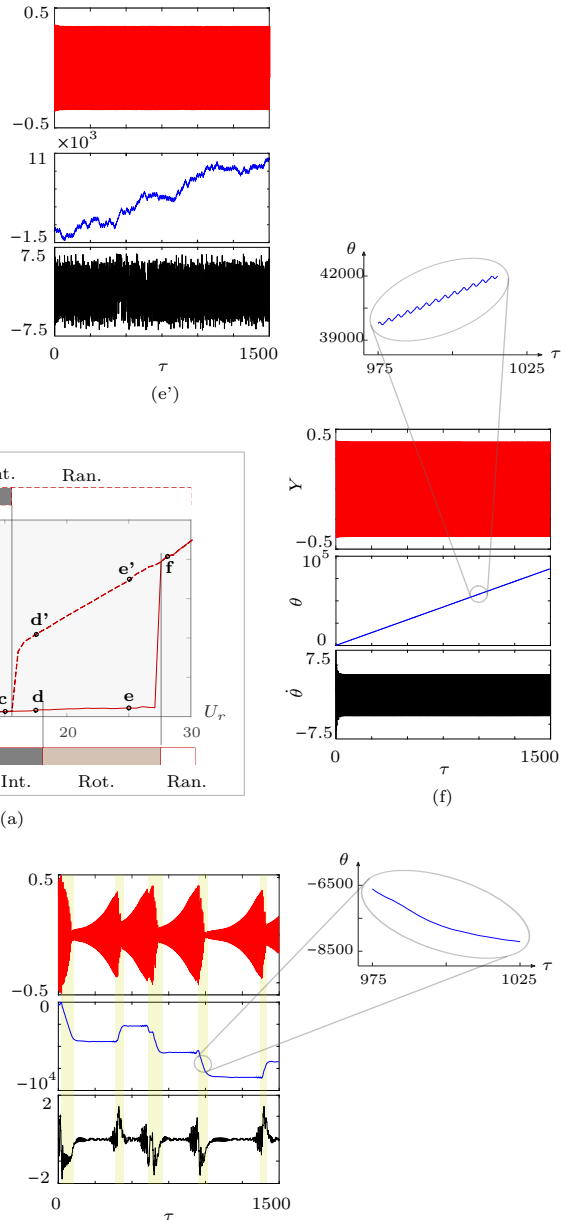

(e)

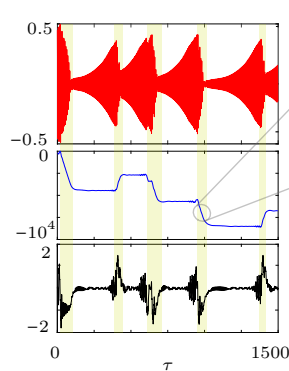

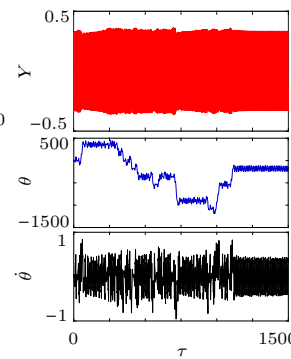

(d)

Fig. 9 Time histories of the prism non-dimensional displacement $Y$ (red curve), NES ball angular displacement $\theta$ in degrees (blue curve), and the the ball angular speed $\dot{\theta}$ (black curve) showing NES dynamics at various flow speeds. (b) Oscillatory, $U_{r}=12.5$; (c, d) Intermittent, $U_{r}=15$ and 17.5, respectively; (e) Rotational, $U_{r}=25$; (f) ineffective regime, $U_{r}=27.5$; (e' and d') are the NES different dynamics observed in case of slowing down the flow to $U_{r}=17.5$ and 25 , respectively. Only the interval $0 \leq \tau \leq 1500$ is shown.

the targeted energy transfer TET mechanism in the prism-NES system, the rotational regime is the most efficient. The NES ball engages with the prism dynamics and rotates with the prism natural frequency in a so-called 1:1 resonance capture phenomenon. That leads to the strongly modulated response of the prism illustrating the nonlinear energy transfer in the prism-NES system. At higher flow velocities, the energy of the prism exceeds the maximum energy absorbed by a ball rotating with the prism natural frequency, which we define as the capacity of the NES. Thus, the ball rotation is ineffective and can not mitigate the galloping amplitudes. 


\section{Analytical estimations}

In this section, we analytically determine the boundary between the NES oscillatory and intermittent regimes by modelling the primary mass as a parametric excitation for the NES. Additionally, a proposed power flow analysis estimates the limit of the NES working range and explains the dynamics of the NES in the rotational regime.

\subsection{Parametric excitations}

Based on the method presented by Gendelman et al (2012), we qualitatively distinguishes between NES different regimes by examining the behaviour of the NES ball from the perspective of a parametric excitation. Assuming a sinusoidal motion for the prism, and substituting in the equation of motion of the rotating ball, equation 10 , we obtain:

$$
\begin{gathered}
Y=Y_{0} \cos \tau, \\
\ddot{\theta}+2 \zeta_{\theta} \dot{\theta}-\frac{Y_{0}}{\hat{r}} \cos \tau \sin \theta=0 .
\end{gathered}
$$

By setting $\ddot{\theta}=\dot{\theta}=0$, we obtain the equilibrium points at $\theta=0, \pi$. The oscillatory regime is characterized by the ball's small oscillations around these points at which $\sin \theta \simeq \theta$. By substituting in equation 12 , we obtain a particular case of the damped Mathieu equation, $\ddot{\theta}+\lambda \dot{\theta}-(\delta+\eta \cos \tau) \theta=0$ (Meirovitch, 2010).

$$
\ddot{\theta}+2 \zeta_{\theta} \dot{\theta}-\frac{Y_{0}}{\hat{r}} \theta \cos \tau=0,
$$

where here $\lambda=2 \zeta_{\theta}, \delta=0$, and $\eta=-\frac{Y_{0}}{\hat{r}}$.

In the previous section, we characterize the oscillatory regime a one with small ball oscillations. Thus, the stability analysis of equation 13 can determine the transition boundary from the oscillatory regime to the intermittent regime; stable equilibrium points indicate the oscillatory regime, while the unstable ones refers to the intermittent regime. The Ince-Strutt diagram representing a stability chart for the Mathieu equation defines the boundaries of parametric instability of equation 9. Based on the expression developed by Kovacic et al (2018) for the boundary of the Ince-Strutt diagram in the case with linear damping $\left(\eta_{\text {crit }}=\frac{1}{2} \sqrt{1+4 \lambda^{2}}\right)$, we can estimate the critical amplitude corresponding to the transition from the oscillatory to the intermittent regime, i.e,

$$
Y_{0_{\text {crit }}}=\hat{r} \sqrt{\frac{1}{4}+4 \zeta_{\theta}^{2}}
$$

where $\zeta_{\theta}$ is the ball damping for small oscillations at low angular speed, = $c / 2 m_{b} r^{2} \omega$. 


\subsection{Power flow analysis}

Numerical results exhibited four different responses for the NES depending on the excitation level. Tracking the energy transfer by the fundamental principles of power flow can explain some critical dynamics for the prism-NES integrated system. We define the non-dimensional power terms of the external and the internal forces affecting our system as the scalar product of the velocity with a given force component: the fluid load power $P_{F y}$, the power of the damping force of the support $P_{d}$, and the power $P_{t r}$ transferred by the NES track reaction on the ball. Besides, terms representing the change in the prism potential energy $\Delta \bar{E}_{p}$ as well as the kinetic energy change due to translation $\Delta \bar{E}_{k_{\text {tran }}}$ and rotation $\Delta \bar{E}_{k_{r o t}}$ are presented. We multiply Eqs. 9 and 10 by $\dot{Y}$ and $\dot{\theta}$ respectively and integrate over a period $\mathrm{T}$ to find

$$
\begin{gathered}
\underbrace{\frac{1}{T} \int_{0}^{T} \frac{\mathrm{d}}{\mathrm{d} \tau}\left[\frac{1}{2} \dot{Y}^{2}\right] \mathrm{d} \tau}_{\Delta \bar{E}_{k_{\text {tran }}}}+\underbrace{\frac{1}{T} \int_{0}^{T} \frac{\mathrm{d}}{\mathrm{d} \tau}\left[\frac{1}{2} Y^{2}\right] \mathrm{d} \tau}_{\Delta \bar{E}_{p}} \\
=\underbrace{\frac{1}{T} \int_{0}^{T} \frac{U_{r}^{2}}{2 m^{*}} C_{y} \dot{Y} d \tau}_{\bar{P}_{F y}}-\underbrace{\frac{1}{T} \int_{0}^{T} 2 \zeta_{y} \dot{Y}^{2} d \tau}_{\bar{P}_{d}}-\underbrace{\frac{1}{T} \int_{0}^{T} \hat{r} \hat{m}\left(\ddot{\theta} \sin \theta+\dot{\theta}^{2} \cos \theta\right) \dot{Y} d \tau,}_{\bar{P}_{t r}} \\
\frac{1}{T} \int_{0}^{T} \hat{m} \hat{r}^{2} \ddot{\theta} \dot{\theta} d \tau=\frac{1}{T} \int_{0}^{T}-2 \hat{m} \hat{r} \zeta_{\theta} \dot{\theta}^{2} d \tau-\frac{1}{T} \int_{0}^{T} \hat{m} \hat{r} \ddot{Y} \sin \theta \dot{\theta} d \tau,
\end{gathered}
$$

We integrate by part the last term of Eq. 16 and rearrange to get

$$
\begin{aligned}
\underbrace{\frac{1}{T} \int_{0}^{T} d\left(\frac{1}{2} \hat{m} \hat{r}^{2} \dot{\theta}^{2}\right)+\frac{1}{T}[\hat{m} \hat{r} \dot{Y} \sin \theta \dot{\theta}]_{0}^{T}}_{\Delta \bar{E}_{k_{r o t}}} \\
=-\underbrace{\frac{1}{T} \int_{0}^{T} 2 \hat{m} \hat{r}^{2} \zeta_{\theta} \dot{\theta}^{2} d \tau}_{\bar{P}_{d i s}}+\underbrace{\frac{1}{T} \int_{0}^{T} \hat{m} \hat{r}\left(\ddot{\theta} \sin \theta+\dot{\theta}^{2} \cos \theta\right) \dot{Y} d \tau}_{\bar{P}_{t r}} .
\end{aligned}
$$

The input power into the prism-NES integrated system $P_{i n}$ and the power transferred to the NES $P_{t r}$ are important parameters informing about the NES effectiveness. The initial stage of the prism response without the NES damping effect represents the NES excitation form. By describing the prism 


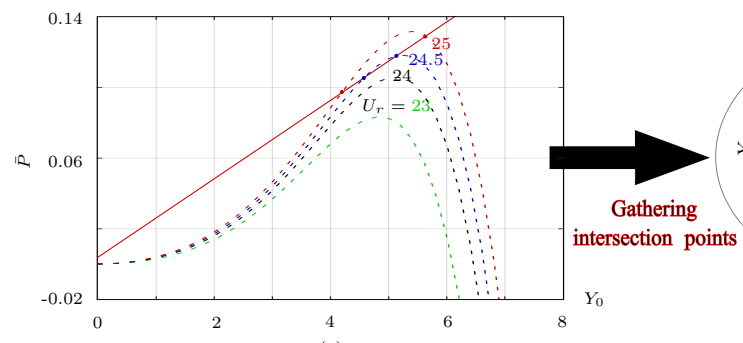

(a)

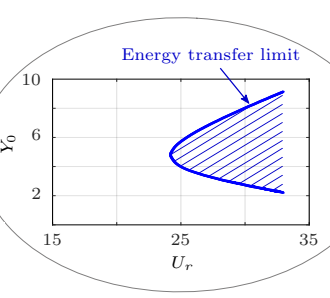

(b)

Fig. 10 The limit of successful energy transfer obtained by (a) comparing the NES maximum power capacity at the rotational regime $\bar{P}_{\text {rot }}(-)$ with the input power $\bar{P}_{\text {in }}$ at various reduced flow velocities: $U_{r}=23(-$ - $), U_{r}=24(--), U_{r}=24.5(--), U_{r}=25$ (- - -). All the averaged-power terms are plotted versus the assumed prism amplitude $Y_{0}$. (b) Energy transfer limit obtained from the intersection of $\bar{P}_{r o t}$ and $\bar{P}_{\text {in }}$ versus $U_{r}$.

displacement in a sinusoidal form (Eq. 11), we estimate $\overline{P_{i n}}$ as follow.

$$
\begin{gathered}
\bar{P}_{i n}=\bar{P}_{F y}+\bar{P}_{d} \\
=\frac{U_{r}^{3}}{4 m^{*}}\left[A_{1}\left(\frac{Y_{0}}{U_{r}}\right)^{2}+\frac{3}{4} A_{2}\left(\frac{Y_{0}}{U_{r}}\right)^{4}+\frac{5}{8} A_{3}\left(\frac{Y_{0}}{U_{r}}\right)^{6}+\frac{35}{64} A_{4}\left(\frac{Y_{0}}{U_{r}}\right)^{8}\right]-Y_{0}^{2} \zeta_{y},
\end{gathered}
$$

where $A_{1}, A_{2}, A_{3}$ and $A_{4}$ are the constants of the $C_{y}$ polynomial fitting presented in Eq. 4. To assess the NES effective range, we compare the averaged value of the input power $\overline{P_{i n}}$ with the NES power capacity. Since the rotational mode represents the most efficient regime in terms of power absorption, at which the ball exhibits a 1:1 resonance capture with the vibrating prism, we estimate the NES maximum power capacity at the rotational regime $\bar{P}_{\text {rot }}$ assuming $\dot{\theta}_{\text {rot }}=1$ and $\theta_{\text {rot }}=\tau+\phi$, where $\phi$ is the phase difference that achieves the maximum transfer rate of energy, which we solved numerically as 0 . By substituting Eq. 11 in the transferred power term presented in Eq.17, $\bar{P}_{\text {rot }}$ can be expressed as

$$
\bar{P}_{\text {rot }}=\frac{1}{2 \pi} \int_{0}^{2 \pi} \hat{m} \hat{r}\left(\ddot{\theta} \sin \theta+\dot{\theta}^{2} \cos \theta\right) \dot{Y} d \tau=\frac{1}{2} \hat{m} \hat{r} Y_{0} .
$$

Comparing the NES maximum power capacity $\bar{P}_{\text {rot }}$ with the input power $\bar{P}_{i n}$ at various flow speeds provides an estimation for the critical reduced flow velocity, at which the NES is no longer effective in absorbing the energy of the galloping prism. Fig. 10 presents the input power at reduced flow velocities $U_{r}=23,24,24.5$, and 25 with the NES maximum power capacity. Since a higher flow speed pumps more energy into the prism-NES system, the input power curve approaches that of the NES capacity by increasing $U_{r}$. The intersection between the $\bar{P}_{i n}$ and $\bar{P}_{\text {rot }}$ curves, observed at $U_{r}=24.5$, indicates that the NES can absorb the input power when it works with its maximum capacity. This defines the critical speed $U_{\text {crit }}$ representing the limit of the NES working 


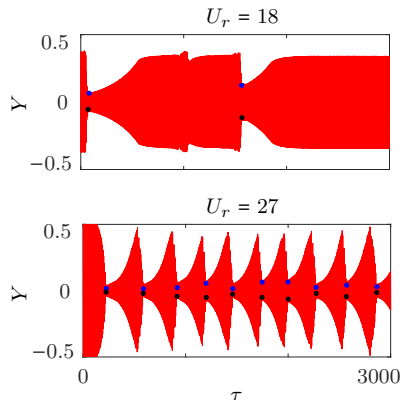

(a)

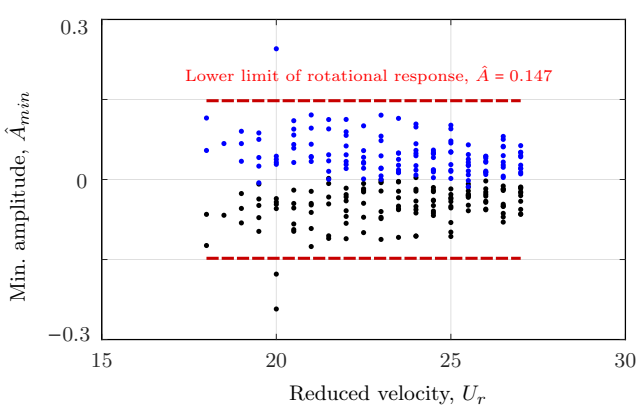

(b)

Fig. 11 The limit of the ball rotational response: (a) time histories of the prism nondimensional displacements $Y(\tau)$ at the beginning $\left(U_{r}=18\right)$ and the end $\left(U_{r}=27\right)$ of the NES rotational regime presenting the minimum amplitudes $\hat{A}_{\text {min }}$ in the $+y(\bullet)$ and $-y(\bullet)$ directions. (b) comparing $\hat{A}_{m i n}$ estimated by simulations at different flow velocities $U_{r}$ with the analytical limit $\hat{A}_{\min }=0.147$ provided by Eq. $21(---)$.

range, beyond which the input power will exceed the NES maximum capacity as occurs at $U_{r}=25$. Gathering the intersection points of $\bar{P}_{\text {rot }}$ curve with $\bar{P}_{i n}$ curves at various flow velocities leads to the predicted limit of successful energy transfer presented in Fig. 10b.

In the perspective of the NES, the transferred power $\bar{P}_{t r}$ is the power gained by the NES, and the power dissipated by ball friction $\bar{P}_{d i s}$ is the power consumed. Consequently, comparing these two values during the ball rotational response $\left(\dot{\theta}_{\text {rot }}=1, \theta_{\text {rot }}=\tau\right)$ can explain the ball behaviour, i.e.,

$$
\begin{gathered}
\bar{P}_{t r}=\bar{P}_{r o t}=\frac{1}{2} \hat{m} \hat{r} Y_{0}, \\
\bar{P}_{d i s}=\frac{1}{2 \pi} \int_{0}^{2 \pi} 2 \hat{m} \hat{r}^{2} \zeta_{\theta} \dot{\theta}^{2} d \tau=2 \hat{m} \hat{r}^{2} \zeta_{\theta} .
\end{gathered}
$$

As long as $\bar{P}_{t r} \geq \bar{P}_{d i s}$, the ball can sustain its rotation to dissipate power until $\bar{P}_{d i s}$ reaches the value of the power in the NES $\bar{P}_{t r}$. When $\bar{P}_{d i s}$ exceeds $\bar{P}_{t r}$, the ball stops rotating. From this comparison, we can get an inequation giving us a minimal limit on the prism amplitude that can sustain the ball:

$$
Y_{0} \geq 4 \hat{r} \zeta_{\theta}
$$

For a NES of $\hat{m}=0.08$ and $\hat{r}=0.6$ as considered here, $4 \hat{r} \zeta_{\theta}=0.147$. Fig. 11 presents the amplitude limit $\hat{A}_{\text {min }}$ below which the ball rotational response is impossible. We defined $\hat{A}_{\text {min }}$ as the minimum prism amplitude observed in each of the periodic cycles of the rotational regime, presented by blue and black points in Fig. 11a. Estimated values of $\hat{A}_{\min }$ obtained from simulations at different flow speeds are almost all below the amplitude limit $\hat{A}_{\text {min }}=0.147$ derived analytically (Fig. 11b). The rotational regime is a competition between the ability of the system to transmit energy from the prism to the ball, and the 


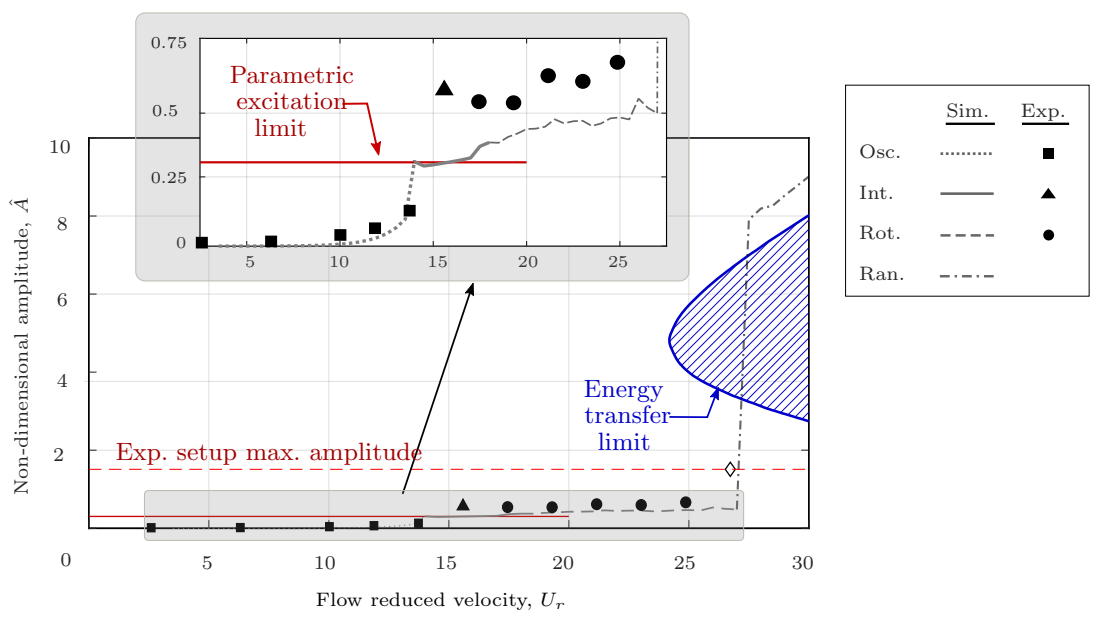

Fig. 12 Physical interruption of our simulation for the prism response with a NES of $\hat{m}=0.08$ and $\hat{r}=0.6$, with experimental results (Selwanis et al, 2021) and analytical estimations (- parametric excitation limit coinciding with the transition between the oscillatory and the intermittent regimes, — the limit of successful energy transfer). The amplitude limitation of the setup is presented by $\diamond$.

capacity of the ball to dissipate energy. The prism accumulates energy from the wind over multiple cycles until the ball is excited, at which point it spins and dissipates energy until the prism amplitude is too small to sustain the motion. This gives rise to the modulated response of the rotating regime (Fig. 9e).

\subsection{Regime Boundaries}

Fig. 12 presents analytical estimations of the boundaries between the NES regimes. Considering the ball in the NES as a parametric excitation problem allows predicting the maximum amplitude below which the oscillatory regime is possible, Eq. 14. This limit is traced as a horizontal red line in Fig. 12. It accurately separates observations of the oscillatory and intermittent regimes, be they experimental or numerical. We can interpret that above a certain amplitude of motion of the prism, the ball undergoes a form of parametric instability and oscillates with great amplitude.

From the power analysis, we can compare the power that can be extracted from the flow by the prism (minus its structural damping) with the maximum energy the ball could dissipate in ideal 1:1 resonance capture where it rotates with a practically constant angular velocity $\dot{\theta}=1$. From this reasoning, we obtain the energy transfer limit curve in blue in Fig. 12. This forms a theoretical limit to the region of effectiveness of the NES. Any imperfection in the functioning of the NES would move this curve to the left as well as make it expand faster. This simple analytical expression found where Eqs.1819 intersect provides a reasonable limit and sheds light on the functioning of the ball-in-track NES for mitigating galloping. 


\section{Conclusion}

The ball-in-track (BIT) NES presented in the literature is a purely rotative NES designed to mitigate the galloping of a square prism in wind tunnel tests. Nevertheless, the limitation in the maximum amplitude of the setup restricted the experiments to a definite range of flow speeds.

The current research proposes a numerical model employing experimental data of the galloping force $C_{y}$ and the ball damping $c_{\theta}$ coefficients as inputs for simulating the dynamics of the prism-NES system. Static experiments provided a fitting for the galloping force coefficient $C_{y}$ as a function of the square prism angle of attack $\alpha$ after eliminating systematic errors using the Kolmogorov-Smirnov (K-S) test. We developed a new method to estimate the ball damping coefficient $c_{\theta}$ through free rotation tests. The experiments provide a critical angular speed, beyond which the ball damping automatically grows due to its contact with both the NES track sidewall and floor. The damping variation is a particular advantage of the BIT-NES; small damping at low angular speeds facilitates the ball's rotation, while relatively large damping allows the ball to absorb more energy at high angular velocities. The simulations exhibit four different responses for the NES ball; oscillatory, intermittent, rotational and ineffective. The oscillatory response of the ball appears at low flow speeds $U_{r}<14$. Within a small range of reduced flow velocities $14<U_{r}<18$, we observe the intermittent regime at which the ball completes a few revolutions with low angular speeds $\dot{\theta}<0.8$. The rotational response seen at higher flow speeds $18<U_{r}<27.5$ is the most efficient regime in terms of energy absorption. In this regime, the NES ball rotates continuously for more than five revolutions, during which it engages with the prism dynamics showing 1:1 resonance capture. Above a critical flow speed $U_{r}>27.5$, the ball experiences an ineffective response with varying speed, and the NES becomes ineffective in reducing galloping amplitudes.

Under a large perturbation or highly energetic initial conditions, the NES can be ineffective in mitigating galloping as the prism vibrates on the upper branch limit cycle. Moreover, the current study provides analytical estimations of the boundaries between NES regimes. Considering the prism mass as a parametric excitation for the NES ball predicts the prism maximum amplitude coinciding with the limit boundary of the oscillatory regime. The proposed power flow analysis across the integrated system of a prism with a BIT-NES of $\hat{m}=0.08$ and $\hat{r}=0.6$ leads to an analytical prediction for the limit of the NES effective range as $U_{r}=24.5$. Power flow analysis in the perspective of the BIT-NES explains the ball dynamics at the rotational regime, pointing out a value of prism amplitude $\hat{A}_{\text {min }} \geq 4 \hat{r} \zeta_{\theta}$ below which the ball rotation stops.

The realistic simulation of the prism-NES dynamics at high flow speeds with the analytical predictions of the NES effective range can help in optimizing the BIT-NES design according to the given parameters of a primary system. That also may extend the practical implementation of such NESs to mitigate vibrations of high rising engineering structures. 
CRediT authorship contribution statement. Michael M. Selwanis: Conceptualization, Methodology, Software, Validation, Formal analysis, Investigation, Writing-original draft, Visualization, Funding acquisition. Guilherme Rosa Franzini: Conceptualization, Methodology, Formal analysis, Writing-review and editing, Funding acquisition. Cédric Béguin: Conceptualization, Methodology, Validation, Formal analysis, Resources, Writing review and editing, Supervision. Frédérick P. Gosselin: Conceptualization, Methodology, Validation, Formal analysis, Resources, Writing-review and editing, Supervision, Project administration, Funding acquisition.

Declarations. The authors declare that they have no known competing financial interests or personal relationships that could have appeared to influence the work reported in this paper.

Acknowledgments. We acknowledge the Military Technical College (MTC) in Egypt and the Natural Sciences and Engineering Research Council of Canada (NSERC) for sponsoring the current project. We thank Bénédict Besner and Nour Aimène, the technicians in the mechanical engineering department of Polytechnique Montreal, for developing the Labview programs and assisting with the design of the measuring system. We are also grateful to Sima Rishmawi for her help in image processing and Souheil Serroud for his assistance during the free rotation tests. The second author acknowledges the Fonds de Recherche du Québec - Nature et technologies (FRQNT), Canada for sponsoring his short-term period as a visiting researcher at Polytechnique Montreal and Brazilian National Council for Scientific and Technological Development (CNPq) for the grant 305945/2020-3.

Supplementary information. Data will be available on request.

\section{Appendix A Eliminating the Systematic Error of Angle Measurement}

We use a protractor read by naked eye to fix the square prism angle of attack expecting a small deviation from the required angle. To eliminate any error that may arise from this deviation, we use the Kolmogorov-Smirnov (K-S) test. This statistical method mainly quantifies how the distribution functions of two data samples are similar. After reflecting $C_{y}$ values measured at negative angles about the origin, we compare it to $C_{y}$ values measured at positive angles (figure A1a). The Kolmogorov-Smirnov test indicates the dissimilarity between the two $C_{y}$ curves by converting the two samples of normal force coefficient data to another form, $C_{y \_}$sum (table A1), where:

$$
\begin{gathered}
C_{y-} \text { sum }_{i}=\sum_{j=1}^{i} C_{y_{j}}, \\
D=\mid \sum_{j=1}^{n}\left(C_{y_{1}-} \text { sum }_{j}-C_{y_{2}-} \text { sum }_{j}\right) \mid .
\end{gathered}
$$




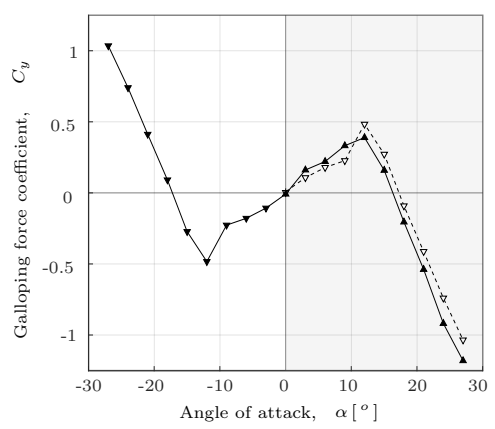

(a)

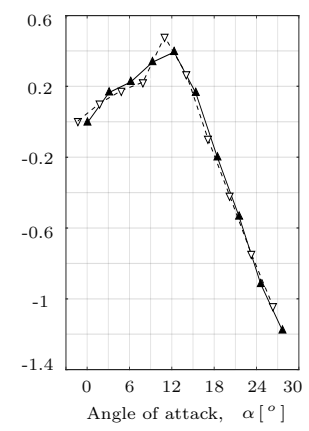

(b)

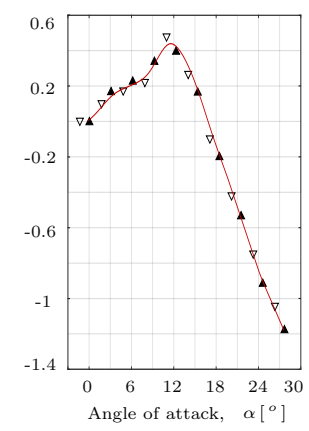

(c)

Fig. A1 The normal force coefficient $C_{y} ; \neg$ measured at positive angles of attack $+\alpha, \rightarrow$ measured at $-\alpha,-\nabla$ - the reflection of the $C_{y}$ curve of negative angles about the origin. (a) direct measurement, (b) measurements shifted by $0.65^{\circ}$ to eliminate the systematic error in the angles' reading, (c) $C_{y}$ fitting (-).

The absolute value of summation of the differences, $D$, indicates how the two data samples follow the same distribution and increases when the data samples are highly dissimilar. For the $C_{y}$ curve, a shift in $\alpha$ changes the value of $C_{y}$ at each angle of attack according to the slope of the curve at this point.

$$
\delta C_{y}=\delta \alpha \cdot \text { slope }
$$

Table A1 K-S test for two sample data of measured $C_{y}$ at the positive and negative angles of attack.

\begin{tabular}{|c|c|c|c|c|c|c|}
\hline$i$ & Angle & $C_{y_{1}}$ & $C_{y_{2}}$ & $C_{y_{1} \_ \text {sum }}$ & $C_{y_{2} \_}$sum & Difference \\
\hline 1 & $0^{\circ}$ & 0.0084 & -0.0084 & 0.0084 & -0.0084 & 0.0168 \\
\hline 2 & $3^{o}$ & 0.1071 & 0.1612 & 0.1155 & 0.1528 & -0.0373 \\
\hline 3 & $6^{o}$ & 0.1789 & 0.2206 & 0.2944 & 0.3734 & -0.079 \\
\hline 4 & $9^{o}$ & 0.2268 & 0.3320 & 0.5212 & 0.7054 & -0.1842 \\
\hline 5 & $12^{\circ}$ & 0.4834 & 0.3891 & 1.0046 & 1.0945 & -0.0899 \\
\hline 6 & $15^{\circ}$ & 0.2722 & 0.1588 & 1.2768 & 1.2533 & 0.0235 \\
\hline 7 & $18^{\circ}$ & -0.0913 & -0.2050 & 1.1855 & 1.0483 & 0.1372 \\
\hline 8 & $21^{o}$ & -0.4123 & -0.5385 & 0.7732 & 0.5098 & 0.2634 \\
\hline 9 & $24^{\circ}$ & -0.7401 & -0.9188 & 0.0331 & -0.4090 & 0.4421 \\
\hline 10 & $27^{\circ}$ & -1.0348 & -1.1807 & -1.0017 & -1.5897 & 0.588 \\
\hline \multicolumn{6}{|c|}{$\mathrm{D}=\sum$ Difference $=$} & 1.08 \\
\hline
\end{tabular}

Based on the Kolmogorov-Smirnov test, a MATLAB code is written to get the optimum shift in $\alpha$ (not exceeding the angle measurement resolution of $\pm 1^{\circ}$ ) that achieves the best symmetry between the measured data of $C_{y}$ at the positive and negative values, see Fig. A1. 
Fig. A1b shows the $C_{y}$ values measured for negative and positive angles of attack with a $0.65^{\circ}$ shift. Using a smoothing parameter $p=0.999995$, a spline fits the measured data after eliminating the systematic error (Fig. A1c). This fitting works as input for the NES-prism mathematical model to introduce accurate values for the galloping force coefficient of the square prism at different angles of attack.

\section{References}

Amar L, Laurendeau E, Michon G, et al (2016) Passive control of flutter applied on a 3DOF airfoil. In: ICTAM 2016-Montréal, Canada

Bearman P, Gartshore I, Maull D, et al (1987) Experiments on flow-induced vibration of a square-section cylinder. Journal of Fluids and Structures 1

Bergeot B, Bellizzi S, Cochelin B (2016) Analysis of steady-state response regimes of a helicopter ground resonance model including a non-linear energy sink attachment. International Journal of Non-Linear Mechanics 78:72-89

Blanchard A, Bergman LA, Vakakis AF (2020) Vortex-induced vibration of a linearly sprung cylinder with an internal rotational nonlinear energy sink in turbulent flow. Nonlinear Dynamics 99(1):593-609

Chen J, Zhang W, Yao M, et al (2018) Vibration reduction in truss core sandwich plate with internal nonlinear energy sink. Composite Structures 193:180-188

Dai HL, Abdelkefi A, Wang L (2016) Usefulness of passive non-linear energy sinks in controlling galloping vibrations. International Journal of Non-Linear Mechanics 81:83-94

Dai HL, Abdelkefi A, Wang L (2017) Vortex-induced vibrations mitigation through a nonlinear energy sink. Communications in Nonlinear Science and Numerical Simulation 42:22-36

Dongyang C, Abbas LK, Guoping W, et al (2018) Numerical study of flowinduced vibrations of cylinders under the action of nonlinear energy sinks (NESs). Nonlinear Dynamics 94:925-957

Franzini GR (2021) An elastic rotative nonlinear vibration absorber (ernva) as a passive suppressor for vortex-induced vibrations. Nonlinear Dynamics 103(1):255-277

Gendelman OV (2001) Transition of energy to a nonlinear localized mode in a highly asymmetric system of two oscillators. Nonlinear dynamics 25(1):237253 
Gendelman OV (2008) Targeted energy transfer in systems with nonpolynomial non linearity. Journal of Sound and Vibration 315:732-745

Gendelman OV, Alloni A (2015) Dynamics of forced system with vibro-impact energy sink. Journal of Sound and Vibration 358:301-314

Gendelman OV, Sigalov G, Manevitch LI, et al (2012) Dynamics of an eccentric rotational nonlinear energy sink. Journal of Applied Mechanics 79:011,012$1-9$

Gomez F, Fermandois GA, Spencer Jr BF (2021) Optimal design of nonlinear energy sinks for mitigation of seismic response on structural systems. Engineering Structures 232:111,756

Guo H, Liu B, Yu Y, et al (2017) Galloping suppression of a suspended cable with wind loading by a nonlinear energy sink. Archive of Applied Mechanics $87: 1007-1018$

Haris A, Alevras P, Mohammadpour M, et al (2020) Design and validation of a nonlinear vibration absorber to attenuate torsional oscillations of propulsion systems. Nonlinear Dynamics pp 1-17

Jin Y, Hou S, Yang T (2021) Cascaded essential nonlinearities for enhanced vibration suppression and energy harvesting. Nonlinear Dynamics pp 1-12

Khazaee M, Khadem S, Moslemi A, et al (2020) A comparative study on optimization of multiple essentially nonlinear isolators attached to a pipe conveying fluid. Mechanical Systems and Signal Processing 141:106,442

Kovacic I, Rand R, Mohamed Sah S (2018) Mathieu's equation and its generalizations: overview of stability charts and their features. Applied Mechanics Reviews $70(2)$

Lee YS, Vakakis AF, Bergman LA, et al (2008) Passive non-linear targeted energy transfer and its applications to vibration absorption: A review. Proceedings of the Institution of Mechanical Engineers, Part K: Journal of Multi-body Dynamics 222:77-134

Li T, Lamarque C, Seguy S, et al (2018) Chaotic characteristic of a linear oscillator coupled with vibro-impact nonlinear energy sink. Nonlinear Dynamics 91:2319-2330

Mehmood A, Nayfeh AH, Hajj MR (2014) Effects of a non-linear energy sink (NES) on vortex-induced vibrations of a circular cylinder. Nonlinear Dynamics 77:667-680

Meirovitch L (2010) Fundamentals of vibrations. Waveland Press 
Menga N, Bottiglione F, Carbone G (2021) Nonlinear viscoelastic isolation for seismic vibration mitigation. Mechanical Systems and Signal Processing

Naudascher E, Weske J, Fey B (1981) Exploratory study on damping of galloping vibrations. Journal of Wind Engineering and Industrial Aerodynamics 8:211-222

Parkinson GV, Smith JD (1964) The square prism as an aeroelastic non linear oscillator. The Quarterly Journal of Mechanics and Applied Mathematics 17:225-239

Pennisi G, Stephan C, Gourc E, et al (2017) Experimental investigation and analytical description of a vibro-impact NES coupled to a single-degree-offreedom linear oscillator harmonically forced. Nonlinear Dynamics 88:17691784

Raj PR, Santhosh B (2019) Parametric study and optimization of linear and nonlinear vibration absorbers combined with piezoelectric energy harvester. International Journal of Mechanical Sciences 152:268-279

Saeed AS, AL-Shudeifat MA, Vakakis AF (2019) Rotary-oscillatory nonlinear energy sink of robust performance. International Journal of Non-Linear Mechanics 117:103,249

Selwanis MM, Franzini GR, Béguin C, et al (2021) Wind tunnel demonstration of galloping mitigation with a purely nonlinear energy sink. Journal of Fluids and Structures 100:103,169

Snoun C, Bergeot B, Berger S (2020) Prediction of the dynamic behavior of an uncertain friction system coupled to nonlinear energy sinks using a multi-element generalized polynomial chaos approach. European Journal of Mechanics-A/Solids 80:103,917

Sun YH, Zhang YW, Ding H, et al (2018) Nonlinear energy sink for a flywheel system vibration reduction. Journal of Sound and Vibration 429:305-324

Teixeira B, Franzini GR, Gosselin F (2018) Passive suppression of transverse galloping using a nonlinear energy sink. Proceeding of the $9^{t h}$ International Symposium on Fluid-Structure Interactions, Flow-Sound Interactions, FlowInduced Vibration and Noise

Tian W, Li Y, Li P, et al (2019) Passive control of nonlinear aeroelasticity in hypersonic 3-d wing with a nonlinear energy sink. Journal of Sound and Vibration 462:114,942 
Tumkur RKR, Domany E, Gendelman OV, et al (2013) Reduced-order model for laminar vortex-induced vibration of a rigid circular cylinder with an internal nonlinear absorber. Communications in Nonlinear Science and Numerical Simulation 18:1916-1930

Ueno T, Franzini GR (2019) Numerical studies on passive suppression of one and two degrees-of-freedom vortex-induced vibrations using a rotative nonlinear vibration absorber. International Journal of Non-Linear Mechanics 116:230-249

Vakakis A (2001) Inducing passive nonlinear energy sinks in vibrating systems. J Vib Acoust 123(3):324-332

Vakakis AF, Gendelman OV, Bergman LA, et al (2008) Nonlinear targeted energy transfer in mechanical and structural systems, vol 156. Springer Science \& Business Media

Wawzonek MA (1979) Aeroelastic behavior of square section prisms in uniform flow. PhD thesis, University of British Columbia

Zhang YW, Hou S, Zhang Z, et al (2020) Nonlinear vibration absorption of laminated composite beams in complex environment. Nonlinear Dynamics pp $1-18$ 